1. Ferrara, N., and Kerbel, R.S. 2005. Angiogenesis as a therapeutic target. Nature. 438:967-974.

2. Mancuso, M.R., et al. 2006. Rapid vascular regrowth in tumors after reversal of VEGF inhibition. J. Clin. Invest. 116:2610-2621. doi:10.1172/JCI24612.

3. Gerber, H.P., and Ferrara, N. 2005. Pharmacology and pharmacodynamics of bevacizumab as monotherapy or in combination with cytotoxic therapy in preclinical studies. Cancer Res. 65:671-680.

4. Kamba, T., et al. 2006. VEGF-dependent plasticity of fenestrated capillaries in the normal adult microvasculature. Am. J. Physiol. Heart Circ. Physiol. 290:H560-H576.

5. Inai, T., et al. 2004. Inhibition of vascular endothelial growth factor (VEGF) signaling in cancer causes loss of endothelial fenestrations, regression of tumor vessels, and appearance of basement membrane ghosts. Am. J. Pathol. 165:35-52.

6. Baluk, P., et al. 2004. Regulated angiogenesis and vascular regression in mice overexpressing vascular endothelial growth factor in airways. Am. J. Pathol. 165:1071-1085.

7. Hu-Lowe, D., et al. 2002. Characterization of potency and activity of the VEGF/PDGF receptor tyrosine kinase inhibitor AG013736. Proc. Am.
Assoc. Cancer Res. 43:A5357.

8. Zou, H.Y., et al. 2004. AG-028262, a novel selective VEGFR tyrosine kinase antagonist that potently inhibits KDR signaling and angiogenesis in vitro and in vivo. Proc. Am. Assoc. Cancer Res. 45:A2578.

9. Conway, E.M., Collen, D., and Carmeliet, P. 2001. Molecular mechanisms of blood vessel growth. Cardiovasc Res. 49:507-521.

10. Kamei, M., et al. 2006. Endothelial tubes assemble from intracellular vacuoles in vivo. Nature. 442:453-456

11. Itoh, T., et al. 1998. Reduced angiogenesis and tumor progression in gelatinase A-deficient mice. Cancer Res. 58:1048-1051.

12. Bergers, G., et al. 2000. Matrix metalloproteinase-9 triggers the angiogenic switch during carcinogenesis. Nat. Cell Biol. 2:737-744.

13. Mott, J.D., and Werb, Z. 2004. Regulation of matrix biology by matrix metalloproteinases. Curr. Opin. Cell Biol. 16:558-564.

14. Stupack, D.G., and Cheresh, D.A. 2004. Integrins and angiogenesis. Curr. Top. Dev. Biol. 64:207-238.

15. Hangai, M., et al. 2002. Matrix metalloproteinase-9 dependent exposure of a cryptic migratory control site in collagen is required before retinal angiogen- esis. Am. J. Pathol. 161:1429-1437.

16. Xu, J., et al. 2001. Proteolytic exposure of a cryptic site within collagen type IV is required for angiogenesis and tumor growth in vivo. J. Cell Biol. 154:1069-1079.

17. Bergsland, E., and Dickler, M.N. 2004. Maximizing the potential of bevacizumab in cancer treatment. Oncologist. 9(Suppl. 1):36-42.

18. Winkler, F., et al. 2004. Kinetics of vascular normalization by VEGFR2 blockade governs brain tumor response to radiation: role of oxygenation, angiopoietin-1, and matrix metalloproteinases. Cancer Cell. 6:553-563.

19. Tong, R.T., et al. 2004. Vascular normalization by vascular endothelial growth factor receptor 2 blockade induces a pressure gradient across the vasculature and improves drug penetration in tumors. Cancer Res. 64:3731-3736.

20. Jain, R.K. 2005. Normalization of tumor vasculature: an emerging concept in antiangiogenic therapy. Science. 307:58-62.

21. Casanovas, O., Hicklin, D.J., Bergers, G., and Hanahan, D. 2005. Drug resistance by evasion of antiangiogenic targeting of VEGF signaling in late-stage pancreatic islet tumors. Cancer Cell. 8:299-309.

\title{
Myeloid suppressor cells regulate the adaptive immune response to cancer
}

\author{
Alan B. Frey \\ Department of Cell Biology, New York University School of Medicine, New York, New York, USA.
}

\begin{abstract}
Inflammation resultant from tumor growth, infection with certain pathogens, or in some cases, trauma, can result in systemic release of cytokines, especially GM-CSF, that in turn stimulate the abundant production and activation of a population of immature myeloid cells, termed myeloid suppressor cells (MSCs), that have potent immunosuppressive functions. In this issue of the JCI, Gallina and colleagues have illuminated some complex issues concerning the development, activation, and function of MSCs (see the related article beginning on page 2777). They show that activation of MSCs is initiated in response to IFN- $\gamma$, presumably produced in situ by antitumor $T$ cells in the tumor microenvironment. After this triggering event, MSCs express 2 enzymes involved in L-arginine metabolism, Arginase I and iNOS, whose metabolic products include diffusible and highly reactive peroxynitrites, the ultimate biochemical mediators of $T$ cell immune suppression. The multifaceted regulation of this complex suppressive effector system provides several potential therapeutic targets.
\end{abstract}

Immune response to tumor growth

As shown elegantly by North, priming of the adaptive immune response occurs during the early stage of tumor growth and results in development of $\mathrm{CD}^{+} \mathrm{T}$ cells reactive to tumors (1). Despite evident host

Nonstandard abbreviations used: IL-4R $\alpha$, IL-4 receptor $\alpha$; L-Arg, L-arginine; MSC, myeloid suppressor cell.

Conflict of interest: The author has declared that no conflict of interest exists.

Citation for this article: J. Clin. Invest. 116:2587-2590 (2006). doi:10.1172/JCI29906. recognition of tumor antigens, coincident with or immediately subsequent to $T$ cell priming, the antitumor immune response is inadequate to eliminate the tumor and is eventually dampened, thereby leading to tumor escape. Understanding how cancer growth affects the antitumor immune response and discovering how escape from antitumor immunity can be reversed are major goals in tumor immunology.

Several non-mutually exclusive phenomena are likely to contribute to inhibition of effective antitumor $\mathrm{T}$ cell immunity, including activation of Tregs (2), cancer "immunoediting" (3), direct tumor downregulation of the effector phase $(4,5)$, and activation of non-T cell suppressor cells (6). Myelopoiesis is often dramatically stimulated during tumor growth, resulting in accumulation (in secondary lymphoid organs, blood, and tumor tissue) and activation of a population of myeloid suppressor cells (MSCs). In mice, removal of the primary tumor results in normalization of the number of systemic MSCs, revealing a causal role for tumor growth in their aberrant accrual (7). Purified MSCs have been shown to inhibit both $\mathrm{CD}^{+}$and $\mathrm{CD}^{+} \mathrm{T}$ cell responses in vitro, and indirect but compelling studies imply that these cells can downregulate $\mathrm{T}$ cell functions in vivo.

\section{MSCs in cancer}

Recent research has focused on deciphering the role played by MSCs in the subversion, inhibition, or downregulation of the immune response to cancer (reviewed in ref. 6). MSCs are uniformly CD $11 \mathrm{~b}^{+} \mathrm{TCR}^{-}$ but express surface markers indicative of a mix of partially differentiated monocytes and DCs. The exact differentiation status of MSCs probably reflects the mechanism 


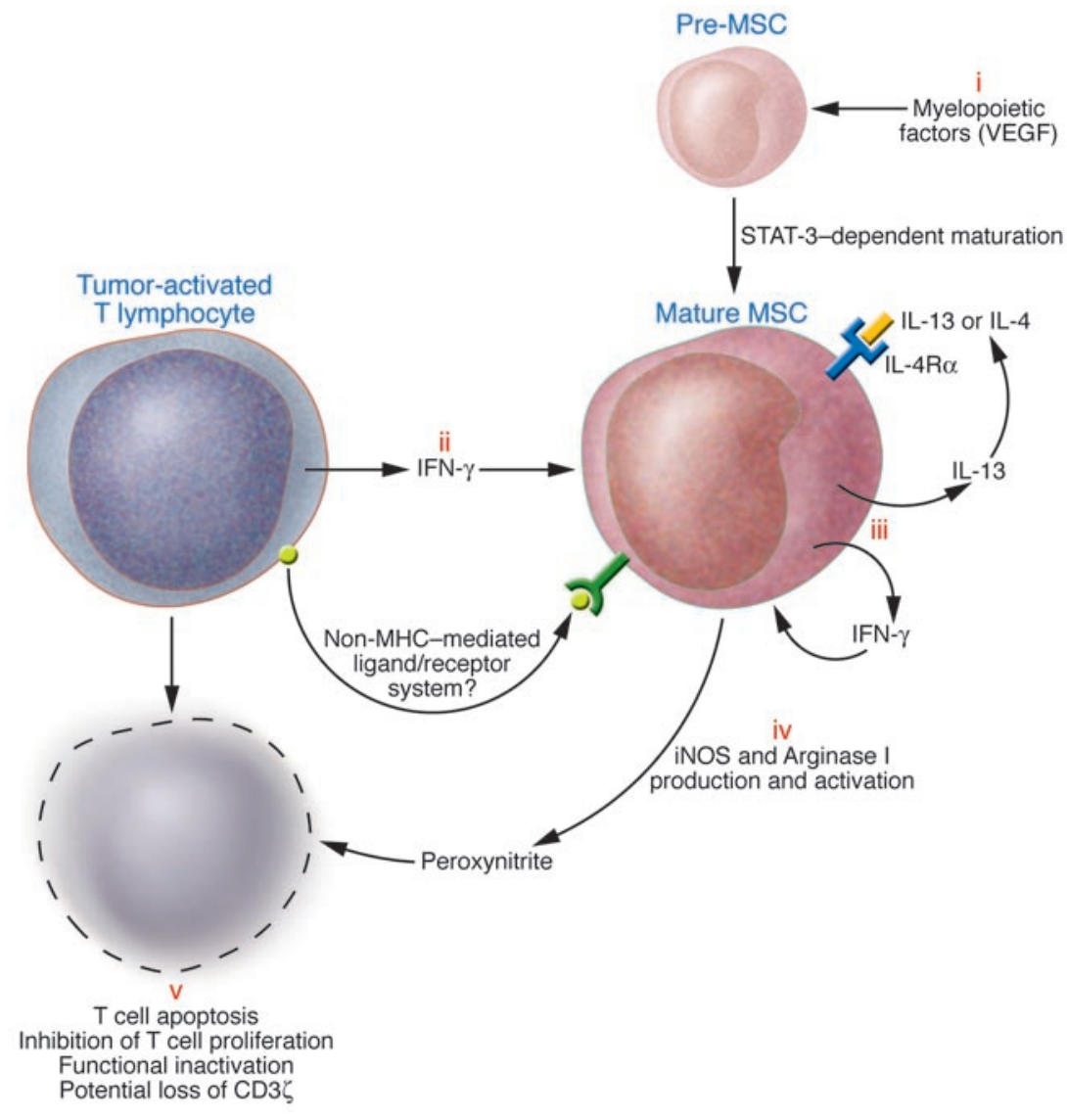

by which their immunosuppressive function may manifest, as discussed below, and is therefore important to determine. However, this is difficult to establish with precision since the function and phenotype of MSCs may vary in different body compartments. Also, as shown by Kusmartsev and colleagues, MSCs are metabolically plastic, evidenced by their ability to differentiate under the influence of select cytokines and differentiation factors into more mature cell types both in vitro and in vivo (8). Significantly, forced maturation of MSCs in vivo was associated with enhancement of vaccination efficacy, suggesting a potentially novel therapeutic strategy that others may reproduce and even expand upon. In an earlier study, deletion of MSCs in tumor-bearing mice using an antibody to the granulocyte marker Gr-1/Ly6G resulted in enhanced immune responses to tumors (9). That report helped to highlight a role for MSCs in regulation of antitumor immune responses in vivo, but use of Ly6 G as a target for MSC depletion may be impossible because this antigen is not necessarily expressed on all functional MSCs. Ly6G is also expressed on neutrophils, which if depleted might confer enhanced susceptibility to microbial infection in patients.

Not only are the differentiation and activation of MSCs incompletely understood, but because MSCs express a veritable cornucopia of pharmacologic agents (including reactive nitrogen and oxygen species, cytokines, proteases, and enzymes important in amino acid metabolism) that can mediate a variety of responses, they have been considered to suppress $\mathrm{T}$ cell immune responses via potentially multiple different mechanisms. The study by Gallina and colleagues in this issue of the JCI has significantly clarified several issues in the MSC field and suggests unanticipated strategies for immunotherapy (10). The authors demonstrate that, upon activation by $\mathrm{CD}^{+} \mathrm{T}$ cell-secreted IFN- $\gamma$, MSCs produce both IL-13 and IFN- $\gamma$, which are utilized in an autocrine manner to enhance the production and activity of 2 enzymes involved in L-arginine (L-Arg) metabolism: iNOS and Arginase I (Figure 1). iNOS produces nitric oxide, which has been shown to interfere with IL-2 receptor signaling such that JAK-1 and -3, STAT-5, and ERK are not activated, leading to cell cycle arrest $(11,12)$. High arginase I activity depletes L-Arg from the

\section{Figure 1}

Immunosuppressive effects of tumor-induced MSCs on antitumor CD8+ T lymphocytes. Previous work has shown that certain factors that enhance myelopoiesis are produced either by tumors or in response to tumor growth (i). In this issue of the $\mathrm{JCl}$, Gallina et al. (10) demonstrate that release of IFN- $\gamma$ by $\mathrm{CD}^{+}$ $\mathrm{T}$ lymphocytes (ii) triggers MSCs to release IL-13 and IFN- $\gamma$ (iii). This results in the production and activation in an autocrine manner of the enzymes iNOS and Arginase I (iv), the downstream effects of which can inhibit $\mathrm{CD}^{+}$ $\mathrm{T}$ cell proliferation and activation or trigger $\mathrm{T}$ cell apoptosis (v). Therapeutic approaches to lifting this MSC-induced immunosuppression during cancer might include targeted deletion of IL-4R $\alpha^{+}$cells (MSCs), blockade of IL-4R $\alpha$, inhibition of STAT-3-dependent pre-MSC maturation into active MSCs, and Arginase I or iNOS inhibition. Figure modified from Gallina et al. (10).

microenvironment and, since activated $\mathrm{CD}^{+} \mathrm{T}$ cells are particularly sensitive to L-Arg levels (13), may result in their functional inactivation. Additionally, it has been shown by the Ochoa lab that CD3 $\zeta$ is lost in T cells exposed to low L-Arg levels (14), a condition postulated to be extant in the tumor microenvironment, which is typically richly infiltrated with MSCs. However, this mechanism put forth to explain dysfunctional tumor-infiltrating lymphocytes has not been observed by others (5, 15-17), and it remains unclear whether $\mathrm{T}$ cell loss of $\mathrm{CD} 3 \zeta$ is restricted to particular types of tumors or to particular types of biochemical analysis.

Another major finding of the current study (10) is that MSCs express IL-4 receptor $\alpha$ (IL-4R $\alpha$ ), an observation that may be useful for the targeted deletion or inactivation of MSCs in vivo. It may also provide an explanation for the observation by Terabe and colleagues that, in murine models, NKT cells producing IL-13 (which shares usage of the IL-4R $\alpha$ with IL-4) caused secretion of TGF- $\beta$ from MSCs and inhibition of antitumor CTLs (18). In their tumor recurrence model, Terabe et al. showed that MSCs produced 2-fold higher TGF- $\beta$ levels in vitro than did nonlymphoid splenocytes isolated from control (non-tumor bearing) mice (18). Administration of neutralizing anti-TGF- $\beta$ protected mice from tumor recurrence, implying a role for TGF- $\beta$ in suppression of antitumor immune responses; the kinetics of the effect suggested a role for TGF- $\beta$ in inhibition of antitumor CTLs. Although they conclude 
the use of a different biochemical mediator of $\mathrm{T}$ cell suppression than Terabe et al., Gallina et al. did not address a role for TGF- $\beta$ in their study, so it is a reasonable possibility that MSCs can utilize multiple biochemical mediators of suppression.

Through the use of strains of mice wherein expression of select cytokines, cytokine receptors, or CD1 was abrogated by gene inactivation, Gallina et al. (10) show that neither NK nor NKT cells are required to elicit or activate MSCs. Although the phenotype of CD1d-restricted NKT cells can vary in strains of distinct genetic backgrounds, favoring Th2 polarization in $\mathrm{BALB} / \mathrm{c}$ and Th1 in C57BL/ 6 mice (19), the disparate results of Gallina et al. and Terabe et al. (18) cannot be explained by strain differences since both studies used BALB/c mice. Thus, the basis for this essential difference remains unclear and may reflect a differential requirement for NKT cells due to different levels of systemic GM-CSF in the 2 models; inherent differences in the nature of the host immune response to the different tumors used; the kinetics of host response such that NKT cells participate in immune suppression at early times of tumor growth but not in later stages; or the fact that Terabe and colleagues strongly stimulated $\mathrm{T}$ cells before measurement of cytokine production (20), whereas Gallina et al. assayed IL-13 and IFN- $\gamma$ production from unstimulated MSCs.

Because expression of Arginase I and iNOS is reciprocally influenced by Th 1 and Th2 cytokines, it has been suggested that activation of each is restricted to separate subsets of myeloid-derived cells, termed alternative activation (21-23). Clearly though, both enzymes are produced in CD $11 b^{+} I L-4 R \alpha^{+}$MSCs, and MSCs produce both Th1-type and Th2-type cytokines (IL-13 and IFN- $\gamma$ ). Therefore, the designation "alternatively activated" may not be applicable or relevant in the characterization of MSCs. More important than the semantic concerns is derivation of the mechanism that MSCs utilize to inactivate $\mathrm{T}$ cell functions. The determination that MSCs produce both Arginase I and iNOS provides insights that can be potentially translated to the clinic. Under conditions of low L-Arg together with activation of iNOS, superoxide is produced (24), which can react with nitric oxide and produce highly reactive peroxynitrites, which can in turn nitrosylate susceptible amino acid side chains, especially tyrosine (25). If proteins important in $\mathrm{T}$ cell signaling are inactivated by nitrosylation, both effector phase functions and differentiation will be abrogated. The demonstration that MSCs exert this complex biochemical scenario, together with the observation that inhibition of Arginase I and iNOS restores T cell function (5), strongly supports the mechanism of MSC function postulated in the current study by Gallina et al. (10). Proof positive will be the identification of $\mathrm{T}$ cell signaling proteins in tumor-infiltrating lymphocytes that are specifically nitrosylated.

\section{Conclusions}

Because at diagnosis patients are often found to have extant MSCs as well as ongoing tumor-induced production of MSCs, immunotherapy of cancer might be improved through downregulation of MSC suppressive functions, which are intended to relieve the inhibition of antitumor $\mathrm{T}$ cell elimination of tumors. Therefore, 2 complementary strategies need to be pursued: blocking the effector mechanism(s) by which MSCs modulate immune responses and blocking the production/activation of MSCs.

There are several potential therapeutic targets for inhibition of MSC function, including IL-13, IL-4R $\alpha$, or either enzyme shown to be essential for peroxynitrite production - Arginase I and iNOS. In this regard, pharmacological inhibitors that function to inhibit iNOS have been tested in tumor models and can be expected to be pursued in further studies (26). In a related study, Arginase I was shown to be induced by prostaglandin $\mathrm{E}_{2}$ that was produced by the tumor (27). Arginase I activity in that model was sensitive to COX-2 inhibition, implying not only another mechanism for induction of immunomodulatory activity by tumors, but also a potential therapeutic opportunity, that of COX-2 inhibition.

Inhibiting the development and/or activation of MSCs might be approached by targeting host- and/or tumor-derived factors that enhance myelopoiesis, for example, GM-CSF. Alternatively, since STAT-3 activation in precursor cells has been shown to be essential for development of MSCs (28), devising schema to inhibit STAT-3dependent pathways in pre-MSCs may prevent their development into fully suppressive MSCs (Figure 1). Additionally, DCs in cancer patients have been shown to be dysfunctional in that their responses to danger signals are blunted (reviewed in ref. 29). Therefore, not only is the ability of intratumoral DCs to prime the immune response potentially compromised, but activation- defective DCs may be tolerogenic, through either direct or indirect involvement in the activation of Tregs $(30,31)$. Targeting STAT-3-controlled differentiation could (hopefully) redirect myelopoiesis such that mature DCs may be produced, instead of the immature DCs that are characteristic of cancer patients, thus enhancing DC priming capacity. Thus, Gallina et al. (10) have unraveled a very complex mechanism of activation and function of suppressor cells that restrict antitumor $\mathrm{T}$ cell immunity.

\section{Acknowledgments}

I thank N. Monu for critical reading of the manuscript and B. Johnson, J. Johnson, and S. Johnson for support.

Address correspondence to: Alan B. Frey, Department of Cell Biology, Room MSB 623, New York University School of Medicine, 550 First Avenue, New York, New York 10016, USA. Phone: (212) 263-8129; Fax: (212) 263-8139; E-mail: freya01@ med.nyu.edu.

1. North, R. 1985. Down-regulation of the antitumor immune response. Adv. Cancer Res. 45:1-42.

2. Yamaguchi, T., and Sakaguchi, S. 2006. Regulatory $\mathrm{T}$ cells in immune surveillance and treatment of cancer. Semin. Cancer Biol. 16:115-123.

3. Dunn, G.P., Bruce, A.T., Ikeda, H., Old, L.J., and Schreiber, R.D. 2002. Cancer immunoediting: from immunosurveillance to tumor escape. Nat. Immunol. 3:991-998.

4. Frey, A.B., and Monu, N. 2006. Effector-phase tolerance: another mechanism of how cancer escapes antitumor immune response. J. Leukoc. Biol. 79:652-662.

5. Bronte, V., et al. 2005. Boosting antitumor responses of T lymphocytes infiltrating human prostate cancers. J. Exp. Med. 201:1257-1268.

6. Serafini, P., Borrello, I., and Bronte, V. 2006. Myeloid suppressor cells in cancer: recruitment, phenotype, properties, and mechanisms of immune suppression. Semin. Cancer Biol. 16:53-65.

7. Salvadori, S., Martinelli, G., and Zier, K. 2000. Resection of solid tumors reverses $T$ cell defects and restores protective immunity. J. Immunol. 164:2214-2220

8. Kusmartsev, S., et al. 2003. All-trans-retinoic acid eliminates immature myeloid cells from tumorbearing mice and improves the effect of vaccination. Cancer Res. 63:4441-4449.

9. Seung, L., Rowley, D., Dubey, P., and Schreiber, H. 1995. Synergy between T-cell immunity and inhibition of paracrine stimulation causes tumor rejection. Proc. Natl. Acad. Sci. U. S. A. 92:6254-6258.

10. Gallina, G., et al. 2006. Tumors induce a subset of inflammatory monocytes with immunosuppressive activity on $\mathrm{CD}^{+} \mathrm{T}$ cells. J. Clin. Invest. 116:2777-2790. doi:10.1172/JCI28828.

11. Bingisser, R.M., Tilbrook, P.A., Holt, P.G., and Kees, U.R. 1998. Macrophage-derived nitric oxide regulates $\mathrm{T}$ cell activation via reversible disruption of the Jak3/STAT5 signaling pathway. J. Immunol. 160:5729-5734.

12. Mazzoni, A., et al. 2002. Myeloid suppressor lines inhibit $\mathrm{T}$ cell responses by an NO-dependent mechanism. J. Immunol. 168:689-695.

13. Bronte, V., Serafini, P., Mazzoni, A., Segal, D.M., 
and Zanovello, P. 2003. L-arginine metabolism in myeloid cells controls T-lymphocyte functions. Trends Immunol. 24:302-306.

14. Rodriguez, P.C., et al. 2004. Arginase I production in the tumor microenvironment by mature myeloid cells inhibits T-cell receptor expression and antigen-specific T-cell responses. Cancer Res. 64:5839-5849.

15. Koneru, M., Schaer, D., Monu, N., Ayala, A., and Frey, A.B. 2005. Defective proximal TCR signaling inhibits CD8+ tumor-infiltrating lymphocyte lytic function. J. Immunol. 174:1830-1840.

16. Franco, J.L., et al. 1995. Partial degradation of T-cell signal transduction molecules by contaminating granulocytes during protein extraction of splenic $\mathrm{T}$ cells from tumor-bearing mice. Cancer Res. 55:3840-3846.

17. Levey, D., and Srivastava, P. 1995. T cells from late tumor-bearing mice express normal levels of p56lck, p59fyn, ZAP-70 and CD3zeta despite suppressed cytolytic activity. J. Exp. Med. 182:1029-1036.

18. Terabe, M., et al. 2003. Transforming growth factor-beta production and myeloid cells are an effector mechanism through which CD1d-restricted T cells block cytotoxic T lymphocyte-mediated tumor immunosurveillance: abrogation prevents tumor recurrence. J. Exp. Med. 198:1741-1752.

19. Hansen, D.S., Siomos, M.A., Buckingham, L., Scalzo, A.A., and Schofield, L. 2003. Regulation of murine cerebral malaria pathogenesis by CD1drestricted NKT cells and the natural killer complex. Immunity. 18:391-402.

20. Terabe, M., et al. 2000. NKT cell-mediated repression of tumor immunosurveillance by IL-13 and the IL-4R-STAT6 pathway. Nat. Immunol. 1:515-520.

21. Gordon, S. 2003. Alternative activation of macrophages. Nat. Rev. Immunol. 3:23-35

22. Mantovani, A., Sozzani, S., Locati, M., Allavena, P., and Sica, A. 2002. Macrophage polarization: tumor-associated macrophages as a paradigm for polarized M2 mononuclear phagocytes. Trends Immunol. 23:549-555.

23. Sinha, P., Clements, V.K., and Ostrand-Rosenberg, S. 2005. Reduction of myeloid-derived suppressor cells and induction of M1 macrophages facilitate the rejection of established metastatic disease. J. Immunol. 174:636-645.

24. Xia, Y., Roman, L.J., Masters, B.S., and Zweier, J.L. 1998. Inducible nitric-oxide synthase generates superoxide from the reductase domain. J. Biol.
Chem. 273:22635-22639.

25. Brito, C., et al. 1999. Peroxynitrite inhibits T lymphocyte activation and proliferation by promoting impairment of tyrosine phosphorylation and peroxynitrite-driven apoptotic death. J. Immunol. 162:3356-3366

26. De Santo, C., et al. 2005. Nitroaspirin corrects immune dysfunction in tumor-bearing hosts and promotes tumor eradication by cancer vaccination. Proc. Natl. Acad. Sci. U. S. A. 102:4185-4190.

27. Rodriguez, P.C., et al. 2005. Arginase I in myeloid suppressor cells is induced by COX-2 in lung carcinoma. J. Exp. Med. 202:931-939.

28. Nefedova, Y., et al. 2004. Hyperactivation of STAT3 is involved in abnormal differentiation of dendritic cells in cancer. J. Immunol. 172:464-474.

29. Gabrilovich, D. 2004. Mechanisms and functional significance of tumour-induced dendritic-cell defects. Nat. Rev. Immunol. 4:941-952.

30. Enk, A.H., Jonuleit, H., Saloga, J., and Knop, J. 1997. Dendritic cells as mediators of tumor-induced tolerance in metastatic melanoma. Int. J. Cancer. 73:309-316.

31. Reis e Sousa, C. 2006. Dendritic cells in a mature age. Nat. Rev. Immunol. 6:476-483.

\title{
To be or not to be B7
}

\author{
Xingxing Zang and James P. Allison
}

Immunology Program, Howard Hughes Medical Institute, Memorial Sloan-Kettering Cancer Center, New York, New York, USA.

\begin{abstract}
The activation of lymphocytes and development of adaptive immune responses is initiated by the engagement of TCRs by antigenic peptide-MHC complexes and shaped at the clonal level by both positive and negative costimulatory signals. The $\mathrm{B} 7$ family members are involved at several stages in this process. In this issue of the JCI, Vogt et al. show that the B7 family-related protein V-set and Ig domain-containing 4 (VSIG4) can act as an inhibitor of $T$ cell activation (see the related article beginning on page 2817). Intriguingly, the same molecule was recently independently identified as a complement receptor of the Ig superfamily (CRIg) and was convincingly demonstrated to be a receptor for complement component 3 fragments. These findings raise interesting questions regarding the physiological roles and mechanisms of action of this molecule. Identification of dual functions of this molecule provides an additional level of complexity in $\mathrm{T}$ cell costimulation.
\end{abstract}

Members of the B7 family provide crucial costimulatory signals that regulate $\mathrm{T}$ cell responses. The major role of the classic costimulatory molecules B7-1, B7-2/CD28, and CTL antigen-4 (CTLA-4) is to regulate $\mathrm{T}$ cell responses at an early stage of $\mathrm{T}$ cell activation in lymphoid tissues. In the past few years,

Nonstandard abbreviations used: C3, complement component 3; CR1, complement receptor 1; CRIg, complement receptor of the Ig superfamily; CTLA-4, CTL antigen-4; PDCD1, programmed cell death 1; VSIG4, $\mathrm{V}$-set and Ig domain-containing 4.

Conflict of interest: The authors have declared that no conflict of interest exists.

Citation for this article: J. Clin. Invest. 116:2590-2593 (2006). doi:10.1172/JCI30103. several new members of the extended B7/CD28 family have been discovered. These include: (a) ICOS ligand (ICOSL; also known as B7h, ICOS-L, B7RP-1, B7-H2) and its receptor ICOS (also known as AILIM, CD278); (b) CD274 (also known as PD-L1, B7-H, B7-H1) and programmed cell death 1 ligand 2 (PDCD1LG2; also known as PD-L2, B7-H2, B7-DC) and their counterreceptor programmed cell death 1 (PDCD1; also known as PD-1); and (c) CD276 (also known as B7-H3, B7RP-2) and $\mathrm{V}$-set domain-containing $\mathrm{T}$ cell activation inhibitor 1 (VTCN1; also known as B7-H4, B7X, B7S1), whose counterreceptors remain unidentified (1). Their precise roles in $\mathrm{T}$ cell regulation are just now being elucidated, but it is apparent that these molecules play important roles in regulating cell-mediated immune responses both temporally and spatially. The blockade of the inhibitory effects of CTLA-4 on T cell proliferation using monoclonal antibodies has been shown to be quite powerful as a means of enhancing immune responses to tumors in mouse studies, and significant clinical effects have been observed in trials of anti-CTLA-4 in melanoma, renal, ovarian, and prostate cancer (2). Similarly, blockade of PDCD1 has been shown to restore the function of exhausted $\mathrm{CD}^{+}$ $\mathrm{T}$ cells during infection and accelerate tumor eradication $(3,4)$. Because of their fundamental biological importance and therapeutic potential, there has been considerable interest in the identification of molecules with costimulatory activity. In this issue of the JCI, Vogt et al. (5) describe a B7 family-related molecule, V-set and Ig domain-containing 4 (VSIG4, also known as Z39Ig [ref. 6] and complement receptor of the Ig superfamily (CRIg) [ref. 7]) and show that it can function as an inhibitor of $\mathrm{T}$ cell activation. Interestingly, a prior study had shown that this molecule was also a complement receptor on macrophages and is required for phagocytosis of circulating pathogens (7). 